\title{
LOWER BOUND FOR THE NORM OF LOWER TRIANGULAR MATRICES ON BLOCK WEIGHTED SEQUENCE SPACES
}

\section{R. LASHKARIPOUR AND G. TALEBI}

Abstract. Let $1<p<\infty$ and $A=\left(a_{n, k}\right)_{n, k \geqslant 1}$ be a non-negative matrix. Denote by $\|A\|_{w, p, F}$, the infimum of those $U$ satisfying the following inequality:

$$
\|A x\|_{w, p, F} \leqslant U\|x\|_{w, p, I},
$$

where $x \geqslant 0$ and $x \in l_{p}(w, I)$ and also $w=\left(w_{n}\right)_{n=1}^{\infty}$ is a decreasing, non-negative sequence of real numbers. The purpose of this paper is to give a lower bound for $\|A\|_{w, p, F}$, where $A$ is a lower triangular matrix. In particular, we apply our results to Weighted mean matrices and Nörlund matrices which recently considered in $[2,3,6]$ on the usual sequence spaces. Our results generalize some work of Jameson, Lashkaripour, Frotannia and Chen in $[4,7,8]$.

Mathematics subject classification (2010): 26D15, 47A30, 40G05, 47D37, 46A45, 54D55.

Keywords and phrases: Norm, upper bound, lower triangular matrix, Nörlund matrices, weighted mean matrices, block weighted sequence space.

\section{REFERENCES}

[1] P. AzImi, A new class of Banach sequence spaces, Bulletin of the Iranian Math. Soc., 28, 2 (2002), $57-68$.

[2] D. Borwein AND F. B. CASs, Nörlund matrices as bounded operators on lp, Arch. Math., 49 (1984), 464-469.

[3] F. B. CASS AND W. KRATS, Nörlund and weighted mean matrices as bounded operators on lp, Rocky Mountain J. Math., 29 (1990), 59-74.

[4] Chang-Pao Chen, Dah-Chin Luor, And Zong-Yin Ou, Extensions of Hardy inequality, J. Math. Anal. Appl., 273 (2002), 160-171.

[5] D. ForOUTANNIA, Upper bound and lower bound for matrix operators on weighted sequence space, Doctoral Dissertation, Zahedan, 2007.

[6] P. D. Johnson JR., R. N. MOHAPATRA AND DAVID RASs, Bounds for the operator norms of some Nörlund matrices, Proc. of the Amer. Math. Soc., 124, 2 (February 1996), 543-547.

[7] G. J. O. JAMESON AND R. LAShKARIPOUR, Norm of certain operators on weighted $l_{p}$ spaces and Lorents sequence spaces, J. Inequalities in Pure Appl. Math., 3, 1 (2002), Article 6.

[8] R. LASHKARIPOUR, D. FOROUTANNIA, Inequlity involving upper bounds for certain matrix operators, Proc. Indian Acad. Sci. (Math. Sci.), 116 (August 2006), 325-336.

[9] R. LashKaripour, D. Foroutannia, Computation of Matrix Operators Bounds with Applying New Extension of Hardy Inequality on Weighted Sequence Spaces I, Lobachevskii Journal of Math., 30, 1 (2009), 40-45. 